\title{
Laimer, Simon, Beschränkung rechtsgeschäftlicher Erfüllungsverpflichtungen.
}

\author{
Mohr Siebeck Verlag, Tübingen, 2020. XXIX, 5511 Seiten. ISBN \\ 978-3-16-155708-8
}

\section{Christian Armbrüster}

Angenommen: 17. Dezember 2020 / Online publiziert: 4. Januar 2021

(C) Der/die Autor(en) 2021

Wer den Titel dieser Neuerscheinung liest, wird kaum ahnen, dass ein Drittel des Buches einem originär versicherungsrechtlichen Thema gewidmet ist, nämlich der Definition des Versicherungsfalls in der Haftpflichtversicherung und deren gerichtlicher Inhalts- und Transparenzkontrolle. Es handelt sich um eine Innsbrucker Habilitationsschrift, die rechtsvergleichend angelegt ist und insbesondere auch die Rechtslage in Deutschland beleuchtet. Dabei geht es um die Auslegung und inhaltliche Gestaltungsgrenzen von vertraglichen Abreden zur Leistungsbegrenzung. Der Autor hat sich dazu zwei Beispielsfälle ausgesucht, nämlich einschränkender Qualitätsvereinbarungen beim Warenkauf (Teil 1, S. 15-308) und eben die formularmäßige Definition des Versicherungsfalls in der Haftpflichtversicherung (Teil 2, S. 309-458). Eingerahmt werden die beiden Beispiele durch eine sehr knapp gehaltene Einleitung (S. 1-13) sowie ein gleichfalls kurzes Resümee (S. 459-463).

Diese Struktur der Arbeit überrascht; man hätte sich eine übergreifende theoretische Grundlegung erhofft, bevor die beiden ausdrücklich also solche bezeichneten Beispiele erörtert werden. Die vom Autor gewählte Struktur führt dazu, dass die Arbeit im Grunde genommen zwei in sich geschlossene eigenständige Monographien enthält, die thematisch miteinander nur locker über den Aspekt der Leistungsbeschränkung verbunden sind. Dessen ungeachtet macht die Auswahl und eingehende Behandlung des zweiten Beispiels - das hier ganz im Vordergrund stehen soll - die Lektüre auch für den versicherungswissenschaftlich ausgerichteten Leser interessant.

$\mathrm{Zu}$ Beginn des dem Versicherungsrecht gewidmeten Teils 2 werden die Grundlagen des Versicherungswesens knapp und präzise dargestellt (S. 309-312). Dies ist für den nicht versicherungsrechtlich vorgebildeten Leser - an den sich das Buch vermutlich in erster Linie richtet - gewiss hilfreich. Sodann kommt Laimer auf die

C. Armbrüster $(\square)$

Fachbereich Rechtswissenschaft, Freie Universität Berlin, Van't-Hoff-Str. 8, 14195 Berlin,

Deutschland

E-Mail: c.armbruester@fu-berlin.de 
einbezogenen Rechtsordnungen zu sprechen. Außer Kontinentaleuropa (Deutschland, Österreich, Italien, Schweiz) und England kündigt er an, auch die Principles of European Insurance Contract Law (PEICL) einzubeziehen. Dies liegt insbesondere im Hinblick darauf nahe, dass die 2. Auflage dieses unverbindlichen Regelwerks auch Bestimmungen zur Haftpflichtversicherung enthält (S. 317, 340). Interessant ist insoweit insbesondere, dass der Versicherungsfalls in Art. 14:107(1) PEICL geregelt und als Kausalereignis definiert wird (S. 340), während der deutsche Gesetzgeber in $\S 100$ VVG eine Festlegung bewusst vermieden hat (s. Regierungsbegr. BT-Drucks. 16/3945, S. 85).

Die anschließende einführende Darlegung zur Haftpflichtversicherung und zu den verschiedenen Definitionen des Versicherungsfalls (S. $320 \mathrm{ff}$., $330 \mathrm{ff}$.) ist akkurat; sie bietet dem Versicherungsrechtler freilich naturgemäß nichts Neues. Was die Anforderungen an die gerichtliche Inhalts- und Transparenzkontrolle angeht, so legt Laimer (S. 354) zutreffend dar, dass aus deutscher Sicht deklaratorische Klauseln, die mit dem Gesetz völlig inhaltsgleich sind (deklaratorische Klauseln im engeren Sinne), nicht kontrollfähig sind. Dies ist insbesondere im Hinblick auf die Transparenzkontrolle nach $\S 307$ Abs. 1 S. 2, Abs. 3 S. 2 BGB bedeutsam, an der wohl nicht wenige gesetzliche Regelungen, unterzöge man sie dieser Kontrolle, scheitern würden. Den entscheidenden Grund für die mangelnde Kontrollfähigkeit, nämlich den anderenfalls eintretenden, mit der Gewaltenteilung gem. Art. 20 Abs. 3 GG unvereinbaren Konflikt zwischen erster und dritter Gewalt, lässt der Autor freilich unerwähnt (s. dazu BGHZ 147, $373=$ NJW 2001, 2012, 2013 [sub I 2 b]; Armbrüster, DNotZ 2004, 437, 443). Dieser Aspekt dürfte für den Fall, dass der europäische Gesetzgeber die PEICL künftig als sog. optionales Instrument autorisiert (s. dazu S. 316), auch bei Art. 2:304 PEICL zur Einschränkung der Kontrollfähigkeit in Bezug auf deklaratorische Klauseln führen. Diese Einschränkung ist nach einer am Wortlaut der Bestimmung orientierten, vom Autor unkommentiert wiedergegebenen Ansicht (S. 400) offenbar nicht vorgesehen.

Akkurat referiert Laimer (S. 366f.) die uneinheitliche Rechtsprechung des BGH zur Kontrollfähigkeit der Versicherungsfalldefinition in der Haftpflicht- und in der Rechtsschutzversicherung. Nach einer Analyse der weiteren in die Untersuchung einbezogenen Rechtsordnungen stellt er fest, dass der von der Inhaltkontrolle ausgenommene Bereich allerorten ,ganz besonders umstritten“ (S. 373) ist. Auch hinsichtlich der Bedeutung der einzelnen Tatbestände zur Inhaltskontrolle ergibt sich rechtsvergleichend ein homogenes Bild: Bei der AVB-Kontrolle steht jeweils die Generalklausel (in Deutschland: $§ 307$ BGB) im Mittelpunkt (S. 387). Regelmäßig führen Verstöße zur Unwirksamkeit der gesamten Klausel, während die Vereinbarung im Übrigen (in Deutschland: nach $\S 306$ BGB) grundsätzlich aufrechterhalten bleibt (S. 398).

Dieses homogene Bild erstaunt angesichts der Vorgaben der europäischen Klauselrichtlinie, von der sich auch das schweizerische Recht inhaltlich nicht weit entfernt, keineswegs. Gelten diese Vorgaben und ihre einzelstaatlichen Umsetzungsgesetze grundsätzlich für alle Vertragsarten, so bezwecken die PEICL einen spezifischen und teils weiter reichenden Schutz des Versicherungsnehmers (S. $398 \mathrm{ff}$.). Dabei ist es unter dem Blickwinkel des Rahmenthemas interessant, dass die Versicherungsfalldefinition zur Haftpflichtversicherung in Art. 14:107 PEICL für die 
Beurteilung der inhaltlichen Angemessenheit abweichender Gestaltungen eine Leitlinie bilden soll (S. 402).

Laimer setzt seine Untersuchung mit der Transparenzkontrolle fort (S. $404 \mathrm{ff}$.). Dabei geht er auf den Umstand, dass der deutsche Gesetzgeber diese Kontrolle im Zuge der Schuldrechtsmodernisierung in $\S 307$ Abs. 1 S. 2 BGB als einen Unterfall der Inhaltskontrolle eingeordnet hat, nicht ein; die Überschrift des gesamten Teils 2 („Gerichtliche Inhalts- und Transparenzkontrolle“) weist vielmehr auf eine klare Trennung hin. Die aus der legislativen Einordnung als Unterfall der Inhaltskontrolle folgende Streitfrage, ob bereits die Intransparenz als solche für eine Unangemessenheit genügt oder ob es darüber hinaus zusätzlich noch einer inhaltlichen Benachteiligung bedarf, bleibt freilich auch bei Laimer (S. $421 \mathrm{f}$.) nicht unerwähnt.

Im Rahmen der Transparenzkontrolle lautet die zentrale Frage, ob der maßgebliche durchschnittliche Versicherungsnehmer ohne versicherungsrechtliche Spezialkenntnisse etwa die Unterscheidung zwischen Schadensereignis und Schadensverursachung zu erkennen vermag. Der Autor zeichnet wiederum die Linien der höchstrichterlichen Rechtsprechung in den maßgeblichen Rechtsordnungen nach. So weist er darauf hin, dass der BGH etwa die Definition des Versicherungsfalls als Schadensereignis i.S. der Folgeereignistheorie in Ziff. 1.1 AHB 2008 für wirksam erachtet hat (S. 412, 452). Interessant sind die Unterschiede zu England und Italien, wo teils strengere Maßstäbe gelten, dies freilich - insoweit den Richtlinienvorgaben entsprechend, also ohne überschießende Umsetzung - allein zugunsten von Verbrauchern (S. 415 ff.). Der Autor geht auch auf die Rechtsfolgen einer Intransparenz ein, die gerade in Deutschland teils lebhaft umstritten sind (S. $421 \mathrm{ff}$.).

Wiederum rundet ein Vergleich mit den PEICL die Betrachtung ab (S. $429 \mathrm{ff}$.). Dabei geht Laimer auch auf den Umstand ein, dass die PEICL keine spezifische Rechtsfolge für die Intransparenz von Klauseln vorsehen (krit. dazu bereits Armbrüster, ZEuP 2008, 775, 782). Soweit er insoweit auf die auch in den PEICL enthaltene Unklarheitenregel (vgl. § 305c Abs. 2 BGB) verweist, ist dem entgegenzuhalten, dass die Transparenzkontrolle von vornherein nur solche objektiv mehrdeutigen Klauseln erfasst, bei denen sich nicht bereits mittels der Unklarheitenregel als einer Auslegungsregel ein eindeutiges Ergebnis ermitteln lässt (eingehend Pilz, Missbräuchliche AGB, 2010, S. 175 ff., 194f.).

Hat die Arbeit bis hierhin weitestgehend darstellenden Charakter, so nimmt Laimer anschließend teils auch Bewertungen vor (S. 433 ff.). Dabei kommt er vorab auf die rechtspolitisch höchst umstrittene Frage zu sprechen, inwiefern die Inhaltskontrolle von AGB im unternehmerischen Geschäftsverkehr sachgerecht ist (S. $435 \mathrm{ff}$.). Den verschiedenen für eine Begrenzung der Kontrollfähigkeit vorgelegten Vorschlägen (eingehend dazu Wendland, Vertragsfreiheit und Vertragsgerechtigkeit, 2019, S. $691 \mathrm{ff}$.) vermag Laimer kaum etwas abzugewinnen. Die Rechtfertigung der AGBInhaltskontrolle erblickt er ,nicht in einer Art von Schwächerenschutz, sondern in dem durch die typische Asymmetrie des Wissensstandes bei Vertragsschluss verursachten partiellen Marktversagen“ (S. 437).

Hier hätte man sich eine intensivere Auseinandersetzung mit den abweichenden Ansätzen gewünscht, zumal die Versicherungsfalldefinition in der Haftpflichtversicherung gerade auch im gewerblichen und industriellen Bereich von großer praktischer Bedeutung ist. Immerhin sieht der Autor (S. 446f.) in $\S 310$ Abs. 1 S. 2 
BGB zutreffend einen möglichen legislativen Anknüpfungspunkt, um de lege lata im unternehmerischen Geschäftsverkehr gewisse Lockerungen gegenüber der am Verbraucherschutz orientierten und in diesem Bereich zu Recht strengen Inhaltskontrolle zu ermöglichen (s. dazu Armbrüster, ZVersWiss 2019, 219, 221 f.).

Vergleichsweise knapp geht Laimer (S. 448 ff.) noch auf die - von ihm kritisch gesehene - Möglichkeit einer geltungserhaltenden Reduktion, auf Einzelfragen des Transparenzgebots und auf die Rechtsfolgen einer Intransparenz ein. Zu Letzteren meint er, dass es für die Missbräuchlichkeit ausreichen dürfte, wenn eine unangemessene Benachteiligung sich daraus ergibt, dass die unklare Formulierung dem Kunden die Wahrnehmung seiner Rechtsposition erschwert; dies sei jedoch nicht von vornherein zu vermuten (S. 455).

Die Ausführungen zum Versicherungsrecht münden in einen Regelungsvorschlag für die Definition des Versicherungsfalls in der Haftpflichtversicherung. Dieser Vorschlag orientiert sich an Art. 14:107(1) PEICL. Er lautet folgendermaßen: „Die Haftpflichtversicherung gilt für Schäden, die während der Versicherungsdauer verursacht worden sind, sofern nicht die Parteien den Versicherungsfall unter Bezugnahme auf andere Kriterien definieren.“ (S. 457). Der Autor meint, dass sich mit dieser Schaffung eines gesetzlichen Leitbildes einige Probleme bewältigen lassen, etwa hinsichtlich des Maßstabs für die Angemessenheitskontrolle abweichender Klauseln und der Lückenfüllung im Fall der Unwirksamkeit einer klauselmäßigen Definition.

Freilich fragt es sich, ob eine einseitige Festlegung gegenüber der derzeitigen Lösung in $\S 100 \mathrm{VVG}$, den Versicherungsfall bewusst nicht festzulegen (BGH VersR 2014, 625 Rn. 34), sachlich gerechtfertigt ist. In der Vertragspraxis haben sich im Laufe der Zeit verschiedenartige Definitionen des Versicherungsfalls herausgebildet, die jeweils den spezifischen Anforderungen des betreffenden Haftpflichtschutzes Rechnung tragen (näher S. $332 \mathrm{ff}$; s. auch Voit, in: Prölss/Martin, VVG, 31. Aufl. 2021, § 100 Rn. 25f.). Wieso sich eine vom Kausalereignisprinzip abweichende Gestaltung an diesem Prinzip als Leitbild messen lassen sollte, leuchtet nicht unmittelbar ein. Dies gilt erst recht im gewerblichen und industriellen Bereich, wo sich teils Sonderformen für bestimmte Versicherungsprodukte wie etwa in der D\&OVersicherung das Claims-made-Prinzip (dazu eingehend Schramm, Das Anspruchserhebungsprinzip, 2009) herausgebildet haben.

Das knappe Resümee, in dem Laimer seine zum Kauf- und zum Versicherungsrecht gefundenen Ergebnisse zusammenfasst und ein Fazit zieht (S. 459-463), bestätigt den bereits aus der Gliederung ersichtlichen Eindruck, dass die beiden Teile seiner Untersuchung weitgehend unverbunden nebeneinander stehen. So lassen sich nur auf hoher Abstraktionsebene Aussagen formulieren, die bereichsübergreifend gelten: Eine asymmetrische Informationsverteilung birgt die Gefahr von Benachteiligungen; das dispositive Gesetzesrecht bietet den Referenzmaßstab für einen gerechten Interessenausgleich; die berechtigten Erwartungen des Kunden sind zu wahren; unterschiedliche Richtlinienumsetzungen erschweren den Binnenmarkt und bringen Rechtsunsicherheit. All dies dürfte unstreitig sein.

Wenn das - gut lesbar verfasste - Buch auch ansonsten kaum mit Widerspruch rechnen muss, liegt dies daran, dass auf die Darstellung von Streitständen und Unterschieden zwischen den einzelstaatlichen Regelungen nur punktuell und auch dann eher zurückhaltend eigene Stellungnahmen des Autors folgen. Seinen eigentlichen 
Wert gewinnt das Werk vielmehr aus der akribischen Zusammenstellung der Rechtslage in den untersuchten Staaten und aus deren Gegenüberstellung mit den - von ihren Verfassern gleichfalls aufgrund rechtsvergleichender Analysen erstellten PEICL. Dieser Wert ist nicht gering zu erachten; die Ausführungen ermöglichen es dem Leser, sich de lege lata wie auch de lege ferenda die verschiedenen Lösungsmöglichkeiten zu streitigen Rechtsfragen zu vergegenwärtigen.

Funding Open Access funding enabled and organized by Projekt DEAL.

Open Access Dieser Artikel wird unter der Creative Commons Namensnennung 4.0 International Lizenz veröffentlicht, welche die Nutzung, Vervielfältigung, Bearbeitung, Verbreitung und Wiedergabe in jeglichem Medium und Format erlaubt, sofern Sie den/die ursprünglichen Autor(en) und die Quelle ordnungsgemäß nennen, einen Link zur Creative Commons Lizenz beifügen und angeben, ob Änderungen vorgenommen wurden.

Die in diesem Artikel enthaltenen Bilder und sonstiges Drittmaterial unterliegen ebenfalls der genannten Creative Commons Lizenz, sofern sich aus der Abbildungslegende nichts anderes ergibt. Sofern das betreffende Material nicht unter der genannten Creative Commons Lizenz steht und die betreffende Handlung nicht nach gesetzlichen Vorschriften erlaubt ist, ist für die oben aufgeführten Weiterverwendungen des Materials die Einwilligung des jeweiligen Rechteinhabers einzuholen.

Weitere Details zur Lizenz entnehmen Sie bitte der Lizenzinformation auf http://creativecommons.org/ licenses/by/4.0/deed.de. 